\title{
A Review on Bacterial Pathogens, Types and Their Inhibition by the Action of Medicinal Plants
}

\author{
Tazeem Zahra ${ }^{1}$, Aleena Alam ${ }^{2}$, Asim Munir ${ }^{1}$, Adnan Shahid ${ }^{1 *}$, Sarfraz Khan ${ }^{1}$, Sahib Jan ${ }^{3}$, Nouman Shoukat ${ }^{1}$ \\ ${ }^{1}$ Institute of Microbiology, University of Agriculture, Faisalabad, Punjab, Pakistan \\ ${ }^{2}$ Department of Botany, University of Agriculture, Faisalabad, Punjab, Pakistan \\ ${ }^{3}$ Department of Clinical Medicine and Surgery (CMS), University of Agriculture, Faisalabad, Punjab, Pakistan
}

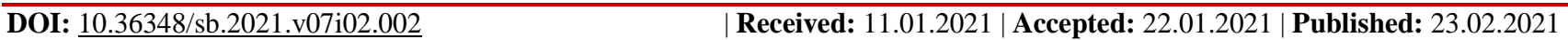

*Corresponding author: Adnan Shahid

\section{Abstract}

Bacterial infections are one cause of food borne illness. Nausea, vomiting, diarrhea, fever, chills, and abdominal pain are common symptoms of food poisoning. Campylobacter jejuni is a diarrheal illness often accompanied by cramps and fever. Clostridium botulinum is a potentially life-threatening bacterium that produces powerful neurotoxins. Escherichia coli is a diarrheal (often bloody) illness that may be accompanied by nausea, vomiting, fever, and abdominal cramps. Listeria monocytogenes causes fever, muscle aches, and diarrhea. Pregnant women, elderly individuals, infants, and those with weakened immune systems are most at risk for acquiring this infection. Salmonella causes fever, diarrhea, and abdominal cramps. Symptoms typically last between 4 and 7 days. Vibrio causes diarrhea when ingested, but it can also cause severe skin infections. Bacterial vaginosis, which causes an overgrowth of pathogenic bacteria in the vagina. Bacterial meningitis is a severe infection of the meninges, the lining of the brain.

Keywords: Antimicrobials, bacterial infections, fever, cramp, food poisoning, antioxidants.

Copyright () 2021 The Author(s): This is an open-access article distributed under the terms of the Creative Commons Attribution 4.0 International License (CC BY-NC 4.0) which permits unrestricted use, distribution, and reproduction in any medium for non-commercial use provided the original author and source are credited.

\section{INTRODUCTION}

Campylobacter jejuni is a genus of bacteria that is among the most common causes of bacterial infections in humans worldwide. Campylobacter means "curved rod", deriving from the Greek kampylos (curved) and baktron (rod). Escherichia coli, also known as E. coli, is a Gram-negative, facultative anaerobic, rod-shaped, coliform bacterium of the genus Escherichia that is commonly found in the lower intestine of warm-blooded organisms [1-3]. Listeria monocytogenes is the species of pathogenic bacteria that causes the infection listeriosis.
It is a facultative anaerobic bacterium, capable of surviving in the presence or absence of oxygen [4].

Salmonella is the type of bacteria that's the most frequently reported cause of food-related illness in the United States. You can't see, smell, or taste it. Illness from these bacteria is officially called salmonellosis. It can cause an upset stomach, diarrhea, fever, and pain and cramping in your belly. Bacterial vaginosis (BV) is an infection of the vagina. It results from a change in the normal balance of vaginal bacteria. BV usually doesn't cause any other health problems. But it can lead to issues, especially when you're pregnant or trying to get pregnant [5]. 


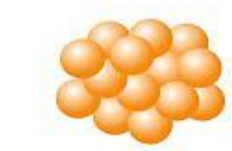

Staphylococcus aureus

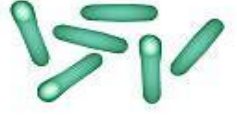

Clostridium botulinum
(ब)

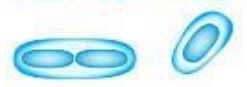

Klebsiella pneumoniae

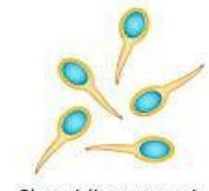

Clostridium tetani

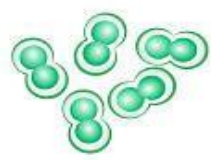

Streptococcus pneumoniae

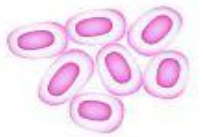

Bordetella pertussis

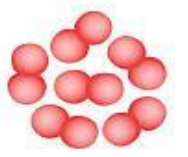

Neisseria gonorrhoeae

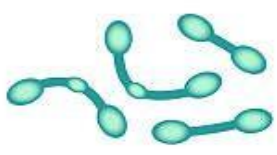

Neisseria gonorrhoeae
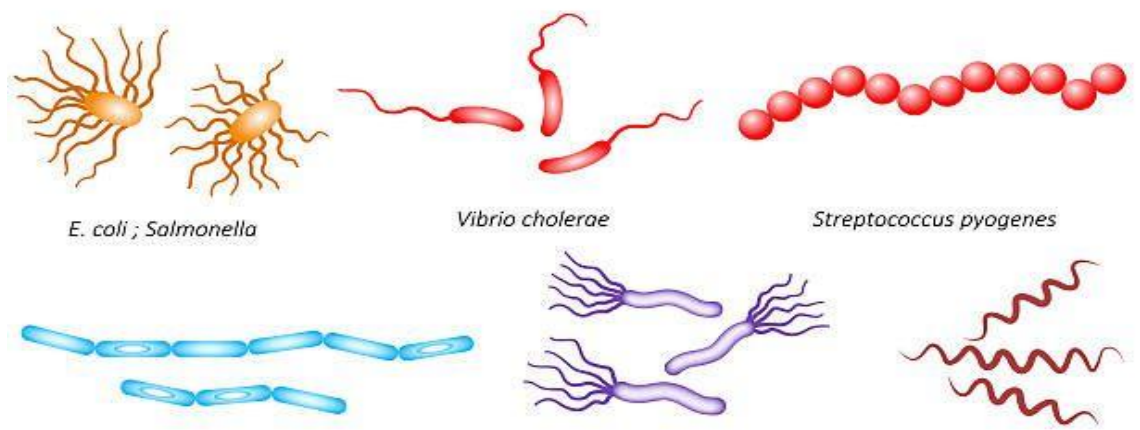

Bacillus cereus

Vibrio cholerae

Streptococcus pyogenes

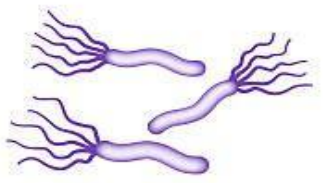

Helicobacter pylori

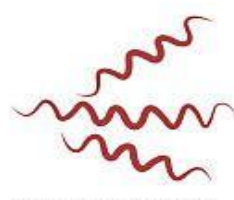

Treponema pallidum

Fig-1: Shows the shapes different types of bacteria

Bacterial meningitis is very serious and can be deadly. Death can occur in as little as a few hours. Most people recover from meningitis. However, permanent disabilities (such as brain damage, hearing loss, and learning disabilities) can result from the infection. Generally, the germs that cause bacterial meningitis spread from one person to another. Certain germs, such as L. monocytogenes, can spread through food [6]. It is also important to know that people can have these bacteria in or on their bodies without being sick. These people are "carriers." Most carriers never become sick, but can still spread the bacteria to others. Most cases of meningitis in the United States are caused by a viral infection, but bacterial, parasitic and fungal infections are other causes. Some cases of meningitis improve without treatment in a few weeks. Others can be lifethreatening and require emergency antibiotic treatment [7-9].

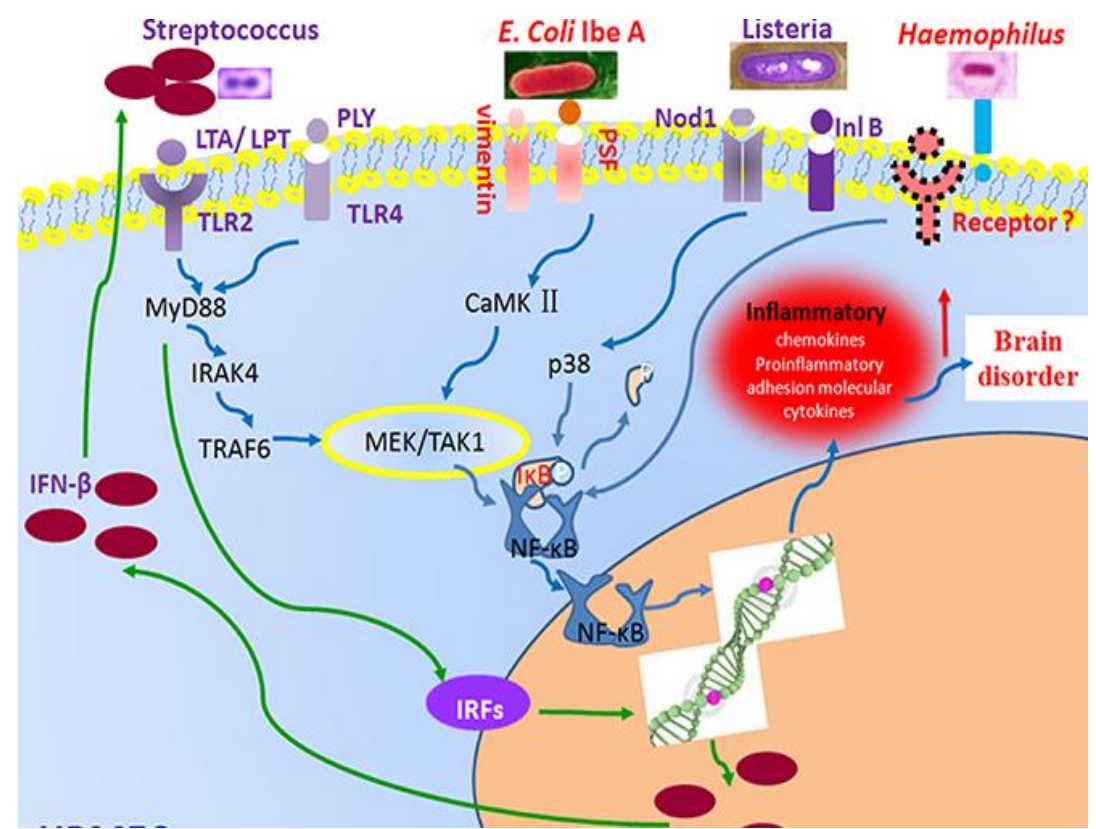

Fig-2: Shows the immune mechanism of different types of bacteria 
Tazeem Zahra et al., Sch Bull, Feb, 2021; 7(2): 9-13

\section{Action of Medicinal Plants for bacterial growth}

Different medicinal plants are widely used as a source of food mostly in industries as well as in homes. But there the certain microbes that causes various diseases in storage of the onions. As a result, the quality of the food reduces which ultimately causes issues to the human health. So, it is important to control the growth of microbes that are harmful and causes diseases in human such as E.coli and S.aureus. These bacteria can cause the spoilage of food and storage of food has becomes issue and needed to resolved. The addition of the certain compounds in the foods as either for their storage as well as the reducing the attacks on the food preserved in industries and homes. Preservatives are compounds that are carefully added in the specific foods to reduce the entry of pathogens as well as bacteria. Their function in the food is to maintain the quality by increasing the shelf life of the selective foods with less or least toxic effects. But the compounds that increasing the shelf life if added more in the quantity as compared to the normal concentration that ultimately leads to the occurrence of many pathogens as well as disease associated with the metabolism [10-12].

Different medicinal plants also exhibit the high potential against the bacteria such as M.luteus. More of the sulfur compounds in the inner portion of the onions and hence strong activity of the onions against the specific type of the bacteria. While less concentration of the cysteine in the onions also leads to the low antimicrobial activity. All compounds in the extracts of the onions possess the activity against the bacteria. Some compounds shows the maximum activity while some has low activity. Coamoxiclave resulted the highest activity against the S.aureus, while the methanol shows the moderate activity and similarly n-hexane shows little activity [13-15].

Different medicinal plants like ginger possess the activity against the different kinds of bacteria such as S.epidermidis as well as the S.aureus. These bacteria involved in causing the diseases associated with the human infections. The data in previous studies proved that the first category of the bacteria such as the S.epidermidis causing the diseases of skin in human. It also damaged the skin by the formation of the lesions that ultimately leads to severe growth of the bacteria. The second category of the bacteria such as S.auerus involved in infections with the urinary system. These bacteria particularly live in the muscles of the urinary system and damaged its wall $[16,17]$.

Previous studies demonstrated that different medicinal plants of possess the activity again the different pathogens like E.coli. This activity depends on the concentration of the antibacterial compounds in the extracts of the onions. The compounds such as the flavonoids shows the highest activity while the other compounds such as the anthocyanin possess the low activity while in inhibiting the growth of the S.aureus. These bacteria shows vigorous changes in behavior when certain concentration extracts applied in vitro based studies. Their growth can be controlled when there are the high concentrations of the flavonoids compounds in the extracts of the plants. Most of the plants such as onions are used as the food by the human and either through the industries [18-21].

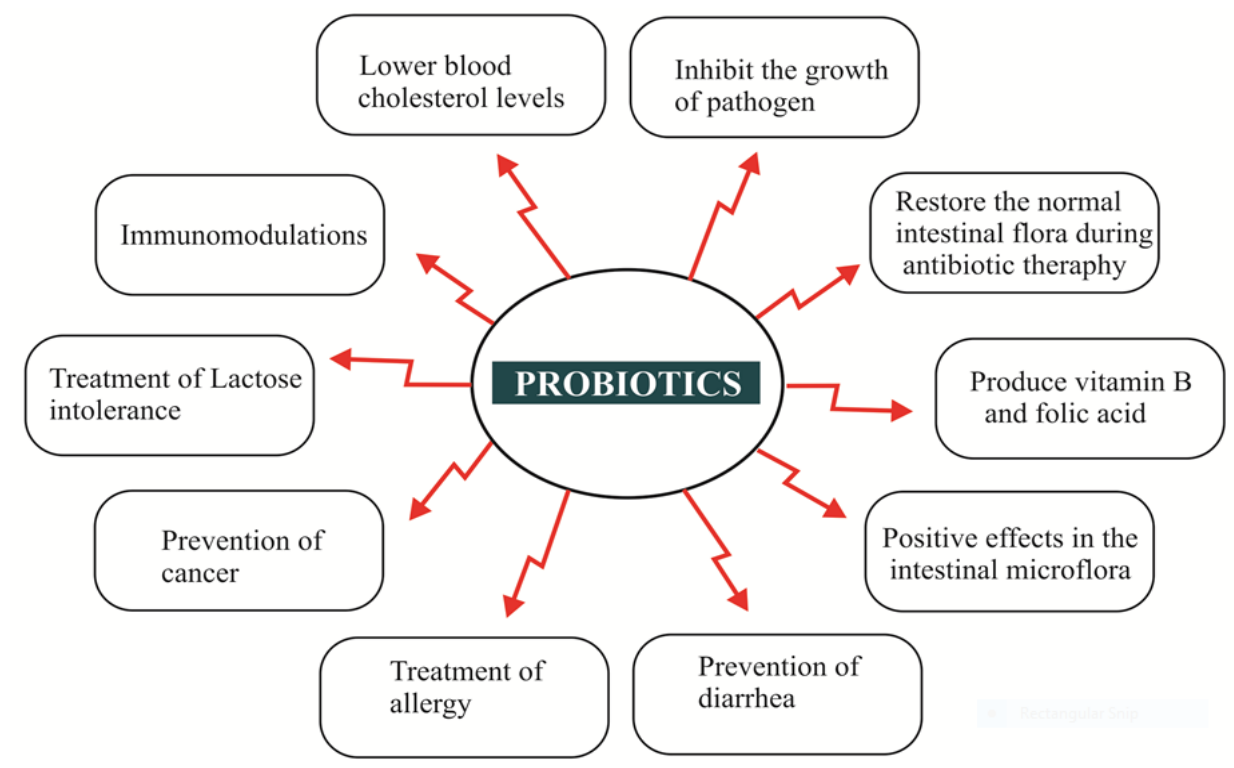

Fig-3: Shows the role of probiotics in prevention of metabolic diereses

\section{Role of Plants antioxidants}

There are various methods for measuring the antioxidant activity of certain compounds in Different medicinal plants as the reaction of the free radicals as well as the antioxidants are very complex. DPPH free radical method is the most important method that is 
used for capturing the free radicals and finally scavenging them. Once the reaction of free radicals started that ultimately leads to the initiation of the chain. It also causes the oxidative stress. To inhibit the chain of the free radicals, antioxidants effectively used to block the chain. For this reason, the antioxidant activity of the certain compounds determined effectively by the DPPH method [21]. Different medicinal plants contains a variety of antioxidants compounds such as phenolics. These phenolic compounds acting as the main source of antioxidants showing the high antioxidant activity. These compounds also protected the DNA by inhibiting the free radicals [22-24].

Antioxidants also inhibited the production of the free radicals. More of the extractions through the methanol ultimately lead to high antioxidant activity while less concentrations of the extraction through the methanol also causes the low antioxidant activity. Extraction through the distill water shows the low potential of the scavenging of the free radicals. Water is used as the source of solvent due to which it has low antioxidant potential as compared to the other organic compounds such as the methanol as well as the nhexane [25].

The antioxidant activity experimentally determined by the transfer of the atom such as hydrogen in DPPH assay. This transfer of hydrogen mainly responsible of antioxidant activity. During the formation of the lipid peroxidation, free radicals such as peroxy radical are causing agents for the blocking of the transfer of the hydrogen atom. This activity only maintained through the transfer of electron that ultimately causes the inhibition of the free radicals. It also reduces the oxidative stress. High potential of the antioxidant activity also inhibited the free radicals and hence more of antioxidants available for the survival of the particular type of the cell [26-29]. Previous studies demonstrated the methanol extract possesses the highest antioxidant activity due to presence of the compounds in different medicinal plants such as phenolic to scavenge the free radicals. The DPPH assay was used in to confirm the correlation the activity to radical scavenge the free radical as well as phenolic [30].

\section{CONCLUSION}

Phenolic compounds are mainly the compounds in medicinal pants that responsible for scavenging the actively free radicals. It also dependent on the concentration of the medicinal plant extracts for the free radicals. The high concentration of the extracts increases the activity to scavenge the free radicals while the low concentration of the extracts decreases the activity to scavenge the free radicals. The extracts of the different medicinal plants utilized as a main source to inhibit the reactions of the oxidation as it contains many of the compounds that concentrated on medicinal plants.

\section{REFERENCES}

1. Shahid, A., Ali, S., Zahra, T., Raza, M., Shahid, A., Saeed, M. U., \& Javaid, F. Influence of Microbes in Progression of Cancer and DNA Damaging Effects.

2. Iftikhar, A., Shahid, A., Shah, S. S., Ali, S., Raza, M., Ali, E., \& Umbreen, S. Antimicrobial Activities of Selected Medicinal Plant with Potential Role of Chemical Compounds.

3. Qamar, M., Mustafa, G. A., Tariq, S., Rafeeq, H., Rafiq, M., Naqvi, W. Z., ... \& Kanwal, T. Novel Methods for Detection of Biological Samples, Current Direction and Future Perspectives.

4. Langford, B. J., So, M., Raybardhan, S., Leung, V., Westwood, D., MacFadden, D. R., ... \& Daneman, N. (2020). Bacterial co-infection and secondary infection in patients with COVID-19: a living rapid review and meta-analysis. Clinical Microbiology and Infection.

5. Yan, L. X., Chen, L. J., Zhao, X., \& Yan, X. P. (2020). pH switchable nanoplatform for in vivo persistent luminescence imaging and precise photothermal therapy of bacterial infection. Advanced Functional Materials, 30(14), 1909042.

6. Hu, W., Chan, H., Lu, L., Wong, K. T., Wong, S. H., Li, M. X., ... \& Zhang, L. (2020, May). Autophagy in intracellular bacterial infection. In Seminars in cell \& developmental biology (Vol. 101, pp. 41-50). Academic Press.

7. Rawson, T. M., Wilson, R. C., \& Holmes, A. (2020). Understanding the role of bacterial and fungal infection in COVID-19. Clinical Microbiology and Infection.

8. Naeem, M., Hayat, M., Qamar, S. A., Mehmood, T., Munir, A., Ahmad, G., ... \& Hussain, A. (2019). Risk factors, genetic mutations and prevention of breast cancer. Int. J. Biosci, 14(4), 492-496.

9. Shafiq, S., Adeel, M., Raza, H., Iqbal, R., Ahmad, Z., Naeem, M., ... \& Azmi, U. R. (2019). Effects of Foliar Application of Selenium in Maize (Zea Mays L.) under Cadmium Toxicity. In Biological Forum-An International Journal (Vol. 11, No. 2, pp. 27-37).

10. Ahmad, I., Khan, S., Naeem, M., Hayat, M., Azmi, U. R., Ahmed, S., ... \& Irfan, M. (2019). Molecular Identification of Ten Palm Species using DNA Fingerprinting. Int. J. Pure App. Biosci, 7(1), 4651.

11. Usman, G., Muhammad, N., Hamza, R., Usman, I., Ayesha, A., Saqib, U., ... \& Fatima, Q. (2019). A Novel Approach towards Nutraceuticals and Biomedical Applications. Scholars International Journal of Biochemistry, 2(10), 245-252.

12. Tahir, M. F., Ali, S., Noman, M., \& Goher, M. A Novel Approach towards the Potential Effects of 
Tazeem Zahra et al., Sch Bull, Feb, 2021; 7(2): 9-13

Chlorpyrifos on Testicular Biochemistry and Physiology of Male Sprague Dawely Rats.

13. Naeem, M., Ali, J., Hassan, M. Z., Arshad, B., Rao, M. H. I., Sarmad, M. S. K., ... \& Hussain, M. U. Novel Approach towards DNA Barcoding as a Tool in Molecular Biology and Biological Activities of Cyclotides with Particular Emphasizes at Molecular Level.

14. Wu, C., Chen, W., He, J., Jin, S., Liu, Y., Yi, Y., ... \& Zhao, W. (2020). Interplay of m6A and H3K27 trimethylation restrains inflammation during bacterial infection. Science advances, 6(34), eaba0647.

15. Wang, H., Zhou, S., Guo, L., Wang, Y., \& Feng, L. (2020). Intelligent Hybrid Hydrogels for Rapid in Situ Detection and Photothermal Therapy of Bacterial Infection. ACS Applied Materials \& Interfaces, 12(35), 39685-39694.

16. Xie, L., Pang, X., Yan, X., Dai, Q., Lin, H., Ye, J., ... \& Chen, X. (2020). Photoacoustic ImagingTrackable Magnetic Microswimmers for Pathogenic Bacterial Infection Treatment. ACS nano, 14(3), 2880-2893.

17. Lopatina, A., Tal, N., \& Sorek, R. (2020). Abortive infection: bacterial suicide as an antiviral immune strategy. Annual Review of Virology, 7, 371-384.

18. Naeem, A., Saddique, S., \& Chand, S. A. (2019). Advancement and Future Directions towards Herbal Treatment for Various Diseases.

19. Naeem, M., Ashraf, A., Safdar, H. M. Z., Khan, M. Q., Rehman, S. U., Iqbal, R., ... \& Ahmad, G. Biochemical changes in patients with chronic kidney failure in relation to complete blood count and anemia.

20. Naeem, M., Hussain, A., Azmi, U. R., Maqsood, S., Imtiaz, U., Ali, H., ... \& Ghani, U. (2019). Comparative Anatomical Studies of Epidermis with Different Stomatal Patterns in Some Selected Plants Using Compound Light Microscopy. International Journal of Scientific and Research Publications, 9(10), 375-380.

21. Ahsan, M., Aslam, M., Akhtar, M. A., Azmi, U. R., Naeem, M., Murtaza, G., ... \& Shafiq, S. (2019). Effect of inoculation of three rhizobial strains on maize hybrids. Journal of Biodiversity and Environmental Sciences, 14(6), 168-177.
22. Hazafa, A., Batool, A., Ahmad, S., Amjad, M., Chaudhry, S. N., Asad, J., ... \& Ghani, U. (2020). Humanin: A mitochondrial-derived peptide in the treatment of apoptosis-related diseases. Life Sciences, 118679.

23. Khan, S., Abbas, A., Ali, I., Arshad, R., Tareen, M. B. K., \& Shah, M. I. (2019). Prevalence of overweight and obesity and lifestyle assessment among school-going children of Multan, Pakistan.

24. Rafeeq, H., Ahmad, S., Tareen, M. B. K., Shahzad, K. A., Bashir, A., Jabeen, R., ... \& Shehzadi, I. Biochemistry of Fat Soluble Vitamins, Sources, Biochemical Functions and Toxicity. Haya: The Saudi Journal of Life Sciences

25. Khan, S., Zelle Rubab, S. H., Abbas, A., Arshad, R., \& Tareen, M. B. K. Hematological profile of children with severe acute malnutrition at the Tertiary care hospital in Multan.

26. Naeem, M., Ashraf, A., Safdar, H. M. Z., Khan, M. Q., Rehman, S. U., Iqbal, R., ... \& Ahmad, G. Biochemical changes in patients with chronic kidney failure in relation to complete blood count and anemia.

27. Kim, T., Zhang, Q., Li, J., Zhang, L., \& Jokerst, J. V. (2018). A gold/silver hybrid nanoparticle for treatment and photoacoustic imaging of bacterial infection. ACS nano, 12(6), 5615-5625.

28. Zhou, J., Yao, D., Qian, Z., Hou, S., Li, L., Jenkins, A. T. A., \& Fan, Y. (2018). Bacteria-responsive intelligent wound dressing: Simultaneous In situ detection and inhibition of bacterial infection for accelerated wound healing. Biomaterials, 161, 1123.

29. Escoll, P., \& Buchrieser, C. (2018). Metabolic reprogramming of host cells upon bacterial infection: Why shift to a Warburg- like metabolism?. The FEBS journal, 285(12), 21462160.

30. Yang, Y., Chu, L., Yang, S., Zhang, H., Qin, L., Guillaume, O., ... \& Tang, T. (2018). Dualfunctional 3D-printed composite scaffold for inhibiting bacterial infection and promoting bone regeneration in infected bone defect models. Acta biomaterialia, 79, 265-275. 